\title{
MACROFAUNA ASSOCIADA A UM CULTIVO SEMI-INTENSIVO DE Litopenaeus vannamei (BOONE, 1931) NO ESTUÁRIO DA LAGOA DOS PATOS (SUL DO BRASIL)
}

\author{
ROSA, L.C. ${ }^{1}$; FREITAS, U. ${ }^{2}$ \& NIENCHESKI, L.F.H ${ }^{3}$ \\ ${ }^{1}$ Centro de Estudos do Mar, Universidade Federal do Paraná (CEM-UFPR). Caixa Postal 50002, 83255- \\ 000, Pontal do Sul, Paraná, Brasil. leonardo.rosa@rocketmail.com \\ 2Instituto Estadual de Meio Ambiente e Recursos Hídricos. Rod. BR 262, Km 0, s/n., 29140-500, \\ Cariacica, Espírito Santo, Brasil. \\ ${ }^{3}$ Laboratório de Hidroquímica, Fundação Universidade Federal do Rio Grande (FURG). Caixa Postal \\ 474, 96201-900, Rio Grande, Rio Grande do Sul, Brasil.
}

\begin{abstract}
Rosa, L.C.; Freitas, U. \& Niencheski, L.F.H. 2009. Macrofauna associated to a semi-intensive Litopenaeus vannamei (Boone, 1931) culture system at the Patos Lagoon estuary (southern Brazil). Braz. J. Aquat. Sci. Technol. 13(2):25-29. ISSN 1808-7035. The macrofauna community structure at two distinct environments (i.e., a pond and a sedimentation basin) of a shrimp farm was compared to the natural environment across entire cycle of Litopenaeus vannamei culture located at estuarine region of the Patos Lagoon, southern Brazil. Both farm habitats showed lower values of number of species and density (pond: $1 \mathrm{spp}$. and 8 ind. $/ 0.008 \mathrm{~m}^{2}$; sedimentary basin: $3 \mathrm{spp}$. and 12 ind. $/ 0.008 \mathrm{~m}^{2}$ ) compared to natural environment (6spp. and 40 ind. $/ 0.008 \mathrm{~m}^{2}$ ). However, the biomass values at sedimentary basin (mean $=0.06 \mathrm{~g} / 0.008 \mathrm{~m}^{2}$ ) were twice higher than natural environment. At pond, low richness of macrofauna seems related to absence of previous pond preparation; while shrimp predation and/or disturbance resulting in low densities after begin of culture. Otherwise, the presence of a biota relatively abundant and with high biomass at sedimentary basin could contribute to increase effluent quality of the shrimp farm.
\end{abstract}

Key-words: Aquaculture; shrimp farm; benthos; community structure

\section{INTRODUÇÃO}

Invertebrados bentônicos desempenham um importante papel nos ambientes de aqüicultura, constituindo um dos principais recursos alimentares naturais nos sistemas de cultivos intensivos e semiintensivos de camarões. Estudos sobre comportamento alimentar de peneídeos em viveiros escavados indicam que $o$ alimento natural pode compreender uma parte significativa da dieta dos camarões, mesmo quando rações peletizadas são fornecidas (Allan et al., 1995; Nunes et al., 1997; Nunes \& Parsons, 1999). Em berçários de Litopenaeus vannamei, Anderson et al. (1987) estimaram que entre $53 \%$ e $77 \%$ do crescimento do camarão foi devido a pastagem sobre a biota do viveiro. Conseqüentemente, o impacto da predação dos camarões sobre a macrofauna pode ser o principal fator controlando suas abundâncias nesses ambientes (Soares et al., 2004).

Por outro lado, a composição e a abundância da comunidade bentônica nesses ambientes de cultivo dependem das técnicas de preparo e de manejo empregadas, podendo estar totalmente ausentes em solos de viveiros pobremente preparados (Shariff et al., 2001).
Além de sua importância trófica, a macrofauna também desempenha um importante papel nos processos de re-mineralização da matéria orgânica e na ciclagem de nutrientes (Mayer et al., 1995; Heilskov \& Holmer, 2001), contribuindo para a manutenção da qualidade físico-química dos solos dos viveiros.

Apesar de sua importância, são poucas as informações sobre a estrutura da comunidade de organismos macrofaunais associada a sistemas de cultivos de camarões marinhos, principalmente aos existentes no Brasil. O objetivo desse estudo foi descrever a estrutura da macrofauna (i.e., composição, riqueza, densidade e biomassa) em dois distintos ambientes (i.e., viveiro e bacia de sedimentação) de um cultivo semi-intensivo de camarões marinhos localizado no estuário da Lagoa dos Patos (sul do Brasil), bem como descrever suas alterações ao longo de um ciclo de produção.

\section{MATERIAL E MÉTODOS}

A Lagoa dos Patos é uma imensa laguna ocupando uma área de aproximadamente $10.360 \mathrm{~km}^{2}$ ao longo da planície costeira do Rio Grande do Sul, 
sendo que somente $10 \%$ de sua área, localizada na porção sul, apresentam características estuarinas (Fig. 1). As características de uma laguna "estrangulada" (sensu Kjerfve, 1986) e a baixa amplitude das marés astronômicas $(<0,4 \mathrm{~m})$ fazem com que o regime de salinidade da região estuarina seja fortemente influenciado pela ação dos ventos e pela taxa de pluviosidade na bacia de drenagem da Lagoa dos Patos (Kantin, 1983; Costa et al., 1998). Períodos de menor pluviosidade, como observados durante o verão e inicio do outono, associados a ventos do quadrante sul favorecem a intrusão da cunha salina ao interior do estuário resultando em elevados valores de salinidade (Rosa \& Bemvenuti, 2006).

O estudo foi realizado na fazenda Carcibrás, situada na porção mediana do estuário (Lat.: $31^{\circ} 55^{\prime}$ 39,20" S; Long.: $052^{\circ} 00^{\prime} 23,31 "$ O) (Fig. 1). A fazenda, primeira do gênero na região, operava na época com três viveiros (de 2 a 4 ha de área e $0,6 \mathrm{~m}$ profundidade cada um) para engorda do camarão marinho Litopenaeus vannamei, onde a densidade média de estocagem foi de 20 indivíduos $/ \mathrm{m}^{2}$, sendo os camarões alimentados com ração peletizada (35\% proteína e 0,7\% fósforo) (Freitas, 2006).

Antes de serem lançados na lagoa, os efluentes oriundos dos viveiros são drenados para uma bacia de sedimentação, distantes aproximadamente $350 \mathrm{~m}$ da margem da laguna (Fig. 1), com o objetivo de reduzir a concentração do material em suspensão.
Nesse estudo foram analisadas as estruturas da macrofauna de dois ambientes distintos: (1) um viveiro de 3,8ha de área e (2) a bacia de sedimentação, sendo que as amostragens foram realizadas durante três etapas, sendo elas no inicio (D0), no meio (D60 $=60$ dias após o início do cultivo) e no final (D120 $=120$ dias depois) de um ciclo de cultivo iniciado em dezembro de 2004. Concomitantemente, a estrutura da macrofauna de uma área rasa próxima ao sistema de cultivo foi analisada e será utilizada numa comparação como sendo o "ambiente de referência". Esses três ambientes serão referidos posteriormente como "Viveiro", "Bacia" e "Lagoa", respectivamente.

Durante cada etapa, seis amostras biológicas foram obtidas com o auxílio de um tubo de PVC $\left(0,008 \mathrm{~m}^{2}\right.$ de área e $20 \mathrm{~cm}$ de profundidade) em cada um dos ambientes, as quais foram peneiradas "in situ" através de uma malha de nylon com abertura de $0,3 \mathrm{~mm}$, sendo o material retido na malha, armazenado em saco plástico devidamente etiquetado e fixado com formalina $10 \%$.

Em laboratório, os organismos foram triados, identificados ao menor grupo taxonômico possível, e quantificados com auxílio de um microscópio estereoscópico. Os valores de biomassa foram estimados através do peso seco, após os organismos terem sidos mantidos em uma estufa a $60^{\circ} \mathrm{C}$ por $48 \mathrm{hs}$ e pesados em uma balança digital $(0,0001 \mathrm{~g}$ de precisão), sendo que as conchas dos moluscos foram dissolvidas com ácido clorídrico $5 \%$ antes da secagem.
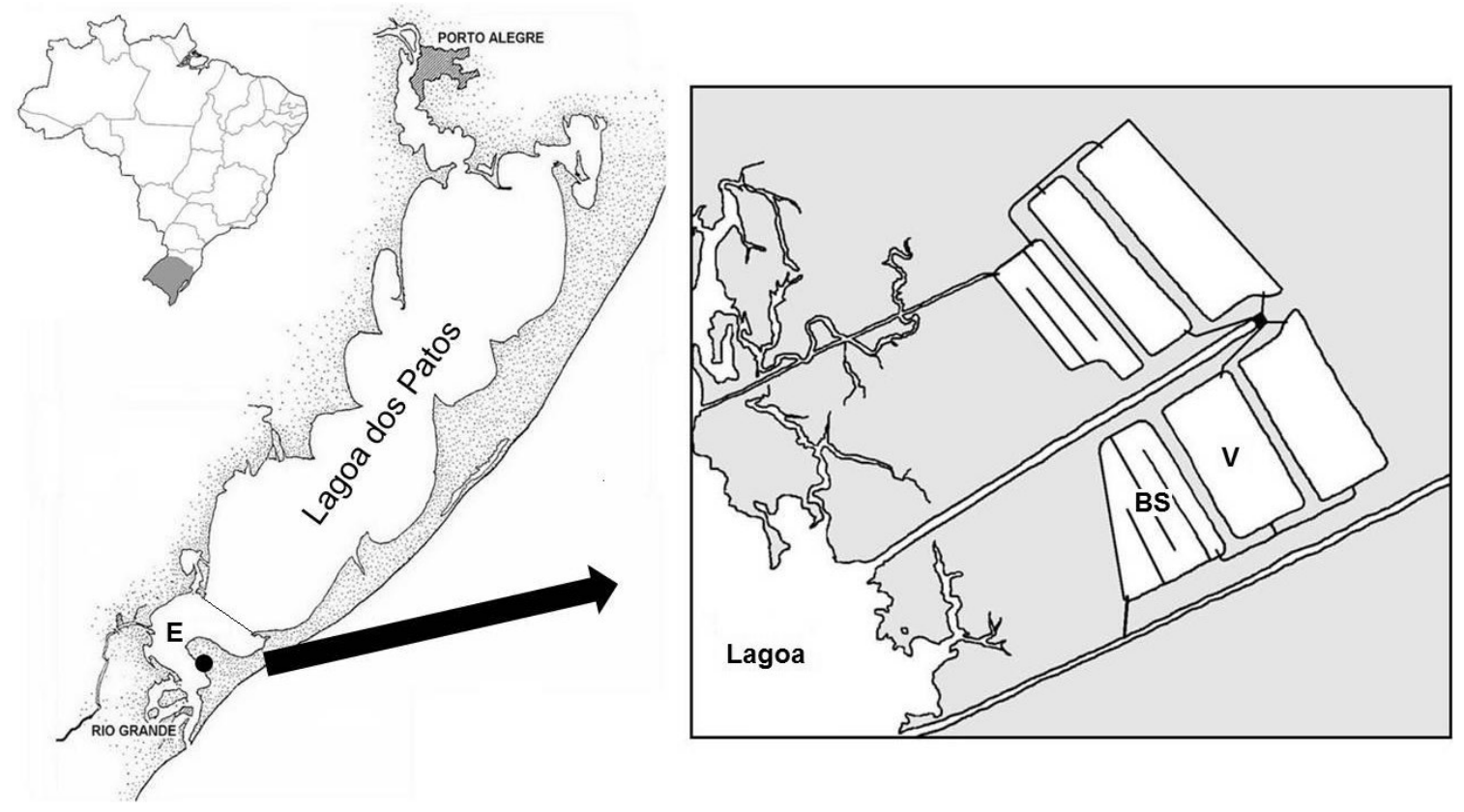

Figura 1 - Mapa da Lagoa dos Patos indicando a localização da fazenda de cultivo de camarão na região estuarina (E) e, no detalhe, a planta da fazenda indicando os ambientes amostrados ( $\mathrm{V}=$ viveiro; $\mathrm{BS}=$ bacia de sedimentação). 


\section{RESULTADOS}

Durante o estudo, foram registrados um total de 13 espécies, sendo que somente cinco destas (Chironomidae, Heleobia australis, Heteromastus similis, Nephtys fluviatilise Laeonereis acuta) ocorreram nos ambientes de cultivo (Tab. 1).

De forma geral, o número de espécies e a densidade da macrofauna foram menores nos ambientes de cultivo comparados ao ambiente natural (Fig. 2a-b). Dos dois ambientes artificiais, o viveiro foi o que apresentou a menor riqueza (i.e., $1 \mathrm{spp}$ ) e os menores valores de densidade (Fig. 2a-b), exceto durante a primeira etapa de amostragem (D0), onde os valores médios de densidade foram similares aos registrados nos outros dois ambientes (Fig. 2b).

Maiores valores de biomassa foram observados na bacia de sedimentação comparada aos outros dois ambientes (Fig. 2c). Tanto na bacia quanto na lagoa, foram registrados um incremento nos valores de biomassa ao longo do cultivo (Fig. 2c)

\section{DISCUSSÃO}

Devido às condições climáticas locais, o período de cultivo do camarão marinho fica restrito aos meses de verão (Freitas, 2006; Poersch et al., 2006), onde além de temperaturas mais elevadas, a baixa pluviosidade favorece maior salinização da água estuarina local devido ao aporte da água marinha (Poersch et al., 2006; Rosa \& Bemvenuti, 2006). Durante o resto do ano, os viveiros permanecem secos, exceto pelo acúmulo ocasional de água da chuva, o que poderia favorecer a ocorrência de organismos límnicos como Chironomidae, os quais foram observados apenas no inicio do cultivo (Tab. 1).
A comunidade da macrofauna estuarina da Lagoa dos Patos é caracterizada por baixa riqueza de espécies (Bemvenuti, 1997), sendo os planos de águas rasas (i.e., $<1 \mathrm{~m}$ ) dominados por organismos infaunais como o tanaidáceo $K$. schubartii e os poliquetas $L$. acuta, $N$. fluviatilis e H. similis (Bemvenuti, 1997; Rosa, 2003; Rosa \& Bemvenuti, 2006). Na região estuarina, essa comunidade apresenta marcada sazonalidade nos padrões de abundância, onde elevadas densidades registradas nas áreas rasas são resultantes do recrutamento de novos indivíduos após intensa atividade reprodutiva desencadeada pelo aumento da temperatura a partir do final da primavera (Bemvenuti, 1997; Rosa, 2003; Rosa \& Bemvenuti, 2006).

O fato do período de produção de camarões marinhos coincidir com o período reprodutivo e, portanto, de intenso recrutamento dos organismos macrofaunais na região, favorece que espécies de desenvolvimento indireto, com uma fase larval planctônica como os poliquetos $L$. acuta, $N$. fluviatilis e $H$. similis possam ser bombeadas para o interior do sistema de cultivo juntamente com a água estuarina. A ausência de $K$. schubartii, um crustáceo peracárido também muito abundante nos planos rasos da região estuarina (Bemvenuti, 1997; Rosa, 2003; Rosa \& Bemvenuti, 2006) e que apresenta uma forma de desenvolvimento direto, corrobora com a hipótese de que organismos macrofaunais colonizam os ambientes de cultivo via coluna d'água, através do processo de bombeamento da água do estuário para dentro dos viveiros.

Depois de assentados, esses organismos sofrem forte influência da predação e/ou perturbação promovida pelos camarões, resultando em baixos valores de densidade da macrofauna como observados no viveiro após o inicio do cultivo (D60 e D120). Os efeitos da predação e/ou perturbação dos camarões sobre a macrofauna é um fato bem documentado na literatura

Tabela 1 - Composição da macrofauna em cada um dos ambientes, ao longo do ciclo de cultivo de camarão Litopenaeus vannamei na região estuarina da Lagoa dos Patos.

\begin{tabular}{|c|c|c|c|c|c|c|c|c|c|}
\hline \multirow{2}{*}{ Espécies } & \multicolumn{3}{|c|}{ Viveiro } & \multicolumn{3}{|c|}{ Bacia } & \multicolumn{3}{|c|}{ Lagoa } \\
\hline & D0 & $\mathrm{D} 60$ & D120 & D0 & $\mathrm{D} 60$ & D120 & D0 & $\mathrm{D} 60$ & D120 \\
\hline \multicolumn{10}{|l|}{ Nemertina } \\
\hline \multicolumn{10}{|l|}{ Heleobia australis (Gastropoda) } \\
\hline \multicolumn{10}{|l|}{ Erodona mactroides (Pelecypoda) } \\
\hline \multicolumn{10}{|l|}{ Tagelus pleibeus (Pelecypoda) } \\
\hline \multicolumn{10}{|l|}{ Heteromastus similis (Polychaeta) } \\
\hline \multicolumn{10}{|l|}{ Nephtys fluviatilis (Polychaeta) } \\
\hline \multicolumn{10}{|l|}{ Laeonereis acuta (Polychaeta) } \\
\hline \multicolumn{10}{|l|}{ Spiophanes sp. (Polychaeta) } \\
\hline \multicolumn{10}{|l|}{ Sphaeromopsis mourei (Isopoda) } \\
\hline \multicolumn{10}{|l|}{ Kalliapseudes schubartii (Tanaidacea) } \\
\hline \multicolumn{10}{|l|}{ Platorchestia monodi (Amphipoda) } \\
\hline \multirow{2}{*}{\multicolumn{10}{|c|}{$\begin{array}{l}\text { Farfantepenaeus paulensis (Penaeidae) } \\
\text { Chironomidae (Insecta) }\end{array}$}} \\
\hline & & & & & & & & & \\
\hline
\end{tabular}


(Allan et al., 1995; Nunes et al., 1997; Shishehchian \& Yusoff, 1999; Soares et al., 2004). Em sistemas intensivos, a densidade da macrofauna nos viveiros normalmente decresce a níveis baixos após cerca de oito semanas de cultivo (Allan et al., 1995; Shishehchian \& Yusoff, 1999). Já, em cultivos realizados em cercados, também no estuário da Lagoa dos Patos, observou-se redução de $85 \%$ na abundância total da macrofauna após um período de 21 dias de cultivo (Soares et al., 2004).

Por outro lado, na bacia de sedimentação, a ausência de predadores e o contínuo aporte de alimento fazem com que os poliquetas adquiram elevada biomassa. Poliquetas são extensivamente utilizados como indicadores de enriquecimento orgânico (Ansari et al., 1986; Clarke \& Warwick, 1994) e suas preferências por ambientes com elevado aporte de material orgânico já havia sido registrado para a região

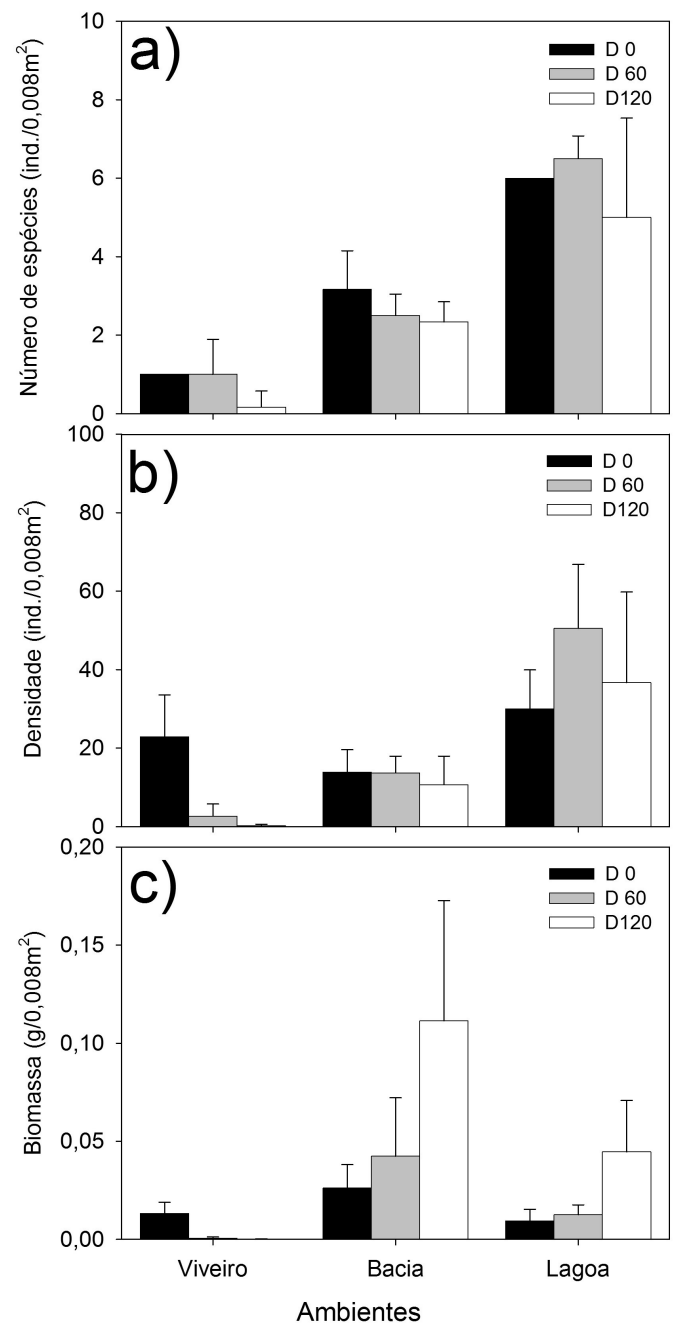

Figura 2 - Valores médios (+ 1 DP) dos parâmetros biológicos medidos em cada ambiente e em cada etapa de coleta. (a) número de espécies, (b) densidade e (c) biomassa da macrofauna. estuarina da Lagoa dos Patos por Angonesi (2000), a qual estudou a influência de um efluente de esgoto doméstico sobre a estrutura da macrofauna estuarina. Segundo a autora, durante o período de recrutamento da macrofauna, elevadas quantidades de indivíduos do poliqueta Laeonereis acuta assentam próximo ao efluente, sugerindo uma preferência desses organismos por locais organicamente enriquecidos.

Nestes locais, os poliquetas alimentam-se da matéria orgânica particulada (Olivier et al., 1995), atuando desta forma na redistribuição das partículas do substrato (Madsen et al., 1997) e na reciclagem dos nutrientes (Mayer et al., 1995), aumentando as taxas de degradação da matéria orgânica e as trocas gasosas entre o substrato e a coluna d'água (Heilskov \& Holmer, 2001), auxiliando desta forma a melhoria da qualidade da água que é lançada de volta à lagoa. No presente caso, o papel de regeneração bêntica desempenhado pela macrofauna bentônica é ainda mais relevante ao considerarmos que dificilmente a bacia de sedimentação é completamente drenada no período de entressafra, o que impede uma adequada degradação da matéria orgânica acumulada no sedimento pela exposição ao sol.

Em conclusão, a baixa riqueza da macrofauna observada no viveiro provavelmente reflete a ausência do emprego de técnicas para estimulação do desenvolvimento da biota nesses ambientes, enquanto que a redução na densidade está associada aos efeitos negativos dos próprios camarões. Por outro lado, abundâncias e biomassas elevadas de poliquetas na bacia de sedimentação podem contribuir para a melhoria da qualidade do substrato e, conseqüentemente, na eficiência do tratamento dos efluentes decorrentes do próprio cultivo.

Apesar das limitações impostas pela ausência de uma replicação adequada, os resultados desse estudo podem ser considerados uma importante contribuição à carcinicultura local, dado a ausência de estudos similares e, principalmente, devido à natureza recente da atividade na região estuarina da Lagoa dos Patos. No entanto, outros estudos deverão ser realizados a fim de confirmar os padrões aqui observados bem como para avaliar melhor o papel da macrofauna nos processos de remineralização da matéria orgânica dentro da bacia de sedimentação.

\section{REFERÊNCIAS}

Allan, G.F.; Moriarty, D.J.W. \& Maguire, G.B. 1995. Effects of pond preparation and feeding rate on production of Penaeus monodon Fabricius, water quality, bacteria and benthos in model farming ponds. Aquaculture. 130: 329-349. 
Anderson, R.K.; Parker, P.L. \& Lawrence, A. 1987. A $13 \mathrm{C} / 12 \mathrm{C}$ tracer study of the utilization of presented feed by a commercially important shrimp Penaeus vannamei in a pond grow out system. J. World Aquacult. Soc. 18: 148-155.

Angonesi, L.G. 2000. Efeitos da descarga de esgotos urbanos sobre os macroinvertebrados bentônicos de fundos moles na região estuarina da Lagoa dos Patos, RS, Brasil. Tese de Mestrado. Fundação Universidade Federal do Rio Grande, Departamento de Oceanografia. 135p.

Ansari, Z.A.; Ingole, B.S. \& Parulekar, A.H. 1986. Effect of high organic enrichment of benthic polychaete population in an estuary. Mar. Pollut. Bull. 17: 361365.

Bemvenuti, C.E. 1997. Benthic Invertebrates. In: Seeliger, U.; Odebrecht, C. \& Castello, J.P. (ed.) Subtropical convergence environments. The coast and sea in the southwestern Atlantic. SpringerVerlag. 43-46pp.

Clarke, K.R. \& Warwick, R.M. 1994. Changes in marine communities: an approach to statistical analyses and interpretation. Natural Environment Research Council, Plymouth, 144p.

Costa, C.S.B.; Seeliger, U. \& Kinas, P.G. 1998. The effect of wind velocity and direction on the salinity regime in the lower Patos Lagoon estuary. Ciênc. Cult. 40: 909-912.

Freitas, U. 2006. Regeneração bêntica e qualidade da água em ambientes sob a ação de cultivos de camarão (Estuário da Lagoa dos Patos). Tese de Mestrado. Fundação Universidade Federal do Rio Grande, Departamento de Oceanografia. 97p.

Heilskov, A.C. \& Holmer, M. 2001. Effects of benthic fauna on organic matter mineralization in fish-farm sediments: importance of size and abundance. ICES J. Mar. Sci. 58: 427-434.

Kantin, R. 1983. Hydrologie et qualité des eaux de la region sud de la Lagune dos Patos (Brasil) et de la plataforme continental adjacente. Tese de Doutorado. Université de Bordeaux I, France. 185p.

Kjerfve, B. 1986. Comparative oceanography of coastal lagoons. In: Wolfe, D.A. (ed.). Estuarine variability. San Diego, Academic Press. 63-81pp.

Madsen, S.D.; Forbes, T.L. \& Forbes, V.E. 1997. Particle mixing by the polychaete Capitella species: I. Coupling fate and effect of a particle-bound organic contaminant (fluoranthene) in a marine sediment. Mar. Ecol. Prog. Ser. 147: 129-142.

Mayer, M.S.; Schaffner, L. \& Kemp, W.M. 1995. Nitrification potentials of benthic macrofaunal tubes and burrow walls: effects of sediment $\mathrm{NH} 4+$ and animal irrigation behavior. Mar. Ecol. Prog. Ser. 121: 157-169.

Nunes, A.J.P.; Gesteira, T.C.V. \& Goddard, S. 1997. Food ingestion and assimilation by the Southern brown shrimp Penaeus subtilis under semi-intensive culture in NE Brazil. Aquaculture. 149: 121-136.

Nunes, A.J.P. \& Parsons, G.J. 1999. Feeding levels of the food dispersal. J. World Aquacult. Soc. 30: 331348.

Olivier, M.; Desrosiers, G.; Caron, A.; Retiere, C. \& Caillou, A. 1995. Behavioral responses of the polychaetes Nereis diversicolor (O.F. Mueller) and Nereis virens (Sars) to food stimuli: use of particulate organic matter (algae and halophytes). Can. J. Zool. 73: 2307-2317.

Poersch, L.; Cavalli, R.O.; Wasielesky-Júnior, W.; Castello, J.P. \& Peixoto, S.R.M. 2006. Perspectivas para o desenvolvimento dos cultivos de camarões marinhos no estuário da Lagoa dos Patos, RS. Cienc. Rural. 36: 1337-1343.

Rosa, L.C. 2003. Variabilidade temporal da estrutura das associações da macrofauna bêntica em uma enseada estuarina da Lagoa dos Patos, RS, Brasil. Tese de Mestrado. Fundação Universidade Federal do Rio Grande, Departamento de Oceanografia. 70p.

Rosa, L.C. \& Bemvenuti, C.E. 2006. Temporal variability of the estuarine macrofauna of the Patos Lagoon, Brazil. Rev. Biol. Mar. Oceanogr. 41: 1-9.

Shariff, M.; Yusoff, F.M.; Devaraja, T.N. \& Srinivasa Rao, P.S. 2001. The effectiveness of a commercial microbial product in poorly prepared tiger shrimp, Penaeus monodon (Fabricius), ponds. Aquac. Res. 32: $181-187$.

Shishehchian, F. \& Yusoff, F.M. 1999. Composition and abundance of macrobenthos in intensive tropical marine shrimp culture ponds. J. World Aquacult. Soc. 30: 128-133.

Soares, R.; Peixoto, S.; Bemvenuti, C.E.; Wasielesky, W.; D'Incao, F.; Murcia, N. \& Suita, S. 2004. Composition and abundance of invertebrate benthic fauna in Farfantepenaeus paulensis culture pens (Patos Lagoon estuary, Southern Brazil). Aquaculture. 239: 199-215. 